\title{
A plate theory for inflatable panels
}

\author{
P. Lacorre*, A. Le van, J.-C. Thomas and R. Bouzidi \\ Institute of Research in Civil and Mechanical Engineering (GeM), CNRS UMR 6183 \\ 2 rue de la Houssinière, BP 92208, 44322 Nantes Cedex 3, France \\ *Corresponding author: paul.lacorre@univ-nantes.fr, web page: https://gem.ec-nantes.fr/
}

\begin{abstract}
Examples of inflatable panels are paddle boards and airbeds. They are membrane structures that acquire load-bearing capacities with internal pressure. They are made of two fabric layers linked together by high-strength threads to keep both sides parallel. The panel is then sealed by lateral walls and pressurized. While it may look like a plate, traditional plate theories cannot be applied since they ignore the internal pressure forces. The aim of this work is to develop a theoretical framework and expertise on inflatable panels to design reliable inflatable buildings.

A theory of inflatable beams has been developed for over 50 years $[1,2,3,4,5]$. However, there is little work on inflatable panels. Wielgosz [6] studied inflatable panels by considering them as beams, but there is no theory of inflatable plates.

In this work, we propose a theoretical model based on the Mindlin-Reissner plate theory which takes into account the internal pressure. The hyperelastic Saint Venant-Kirchhoff material model is used for the membranes. Nonlinear equations for the dynamics of inflatable panels are derived from the principle of virtual power for large displacements. The resulting PDEs can be solved for the displacement of the mid-surface and the direction of the fibers. In order to find analytical solutions, we linearized around the pre-stressed equilibrium state.

Solutions are found for clamped or simply-supported circular panels with uniform vertical load, as well as for rectangular panels with supported and free edges. The pressurization gives rise to additional stiffness terms which play a key part in structural integrity. The analytical solutions are compared to 3D finite element results. In the linear elastic range, the predicted deflection is found to be in good accordance with finite element simulations.
\end{abstract}

\section{REFERENCES}

[1] R.L. Comer and S. Levy, Deflections of an inflated circular-cylindrical cantilever beam, AIAA journal, Vol. 1(7), 1652-1655 (1963).

[2] J.P.H. Webber, Deflections of inflated cylindrical cantilever beams subjected to bending and torsion, Aeronautical Journal, Vol. 86(858), 306-312 (1982).

[3] W.B. Fichter, A theory for inflated thin-wall cylindrical beams, NASA Technical Note, NASA TND3466; (1966).

[4] A. Le van and C. Wielgosz, Bending and buckling of inflatable beams: some new theoretical results, Thin-Walled Structures, Vol. 43(8), 1166-1187 (2005).

[5] Q.T. Nguyen and J.-C. Thomas and A. Le van, Inflation and bending of an orthotropic inflatable beam, Thin-Walled Structures, Vol. 88, 129-144 (2015).

[6] C. Wielgosz and J.-C. Thomas, Deflection of inflatable panels at high pressure, Thin-Walled Structures, Vol. 40(6), 523-536 (2002). 